\title{
Cestocidal effects of Balanites aegyptiaca fruits' methanolic extract on the adult Dipylidium caninum
}

\author{
Hatem A. Shalaby*, Omnia M. Kandil, Noha M. Hassan and Mohamed A. Helal
}

\begin{abstract}
Background: The persistent problem of canine infection with Dipylidium caninum, in spite of the wide accessibility of drugs proposed essentially to kill cestodes, recommends the need to discover other effective alternative therapy. The present study aimed to explore the role of Balanites aegyptiaca fruits against the adult $D$. caninum, and whether its methanolic extract had any effect on the tegument of adult cestode which is essential for the protective function, following 12- and 24-h incubation in vitro. The tegumental alterations were assessed by using both light and scanning electron microscopic studies.

Results: The Balanites extract exhibited cestocidal effect in vitro on D. caninum, and the tegument of the adult worm appeared to be its primary target. This finding was supported by differences in extract response depending on the exposure period. After 12-h incubation in vitro with Balanites extract, the tegumental changes concerned the scolex other than the proglottides, while by $24-\mathrm{h}$ incubation, the changes in the adult cestodes concerned the whole body surface.

Conclusion: The tegumental distortion appeared to be the main changes induced by treatment with the tested extract. This alteration would obviously disrupt the protective function of the tegument and facilitate expelling of the adult cestode from the dog's intestine.
\end{abstract}

Keywords: Dipylidium caninum, Balanites aegyptiaca, Fruits, Cestocidal, In vitro effects

\section{Introduction}

Helminthic infection is the most common disease in dogs everywhere in the world. Despite the accessibility of successful medications to treat helminthes, most helminthes of dogs have exceptionally advanced life cycles that make their disposal unthinkable. Besides, dogs are routinely infected with gastrointestinal helminthes, sometimes without obvious evidence of the infection. Dipylidium caninum is the most common tapeworm of dogs that requires the participation of an arthropod in its life cycle. It is transmitted by ingesting an intermediate host, flea, which carries the larval forms or cysticercoids. The infected dogs shed proglottids in their feces, and these

\footnotetext{
* Correspondence: shalaby85@gmail.com

Department of Parasitology and Animal Diseases, National Research Centre, P.O. Box 12622, Giza, Egypt
}

\section{Springer Open}

proglottids in the environment are consumed by flea larvae (Wani et al. 2015). Human infections have also been reported from many areas of the world and usually identified in children 1 to 5 years old (Szwaja et al. 2011). In spite of the wide accessibility of effective ectoparasiticides which ought to eliminate exposure to infected fleas, canine infection with $D$. caninum keeps on being to be a persistent problem (Barnett et al. 2013). This disease in canines has been generally treated with drugs proposed essentially to kill cestodes, for instance, praziquantel, pyrantel, and oxantel (Grandemange et al. 2007). Yet, there are helminthes that showed resistance to some of these drugs (Kopp et al. 2007). Moreover, experimental studies have shown that these drugs have side effects such as nausea, vomiting, and hepatomegaly (Yangco et al. 1987), which recommends the need to discover other effective 
alternative therapy that is safe and economical. A number of medicinal plants are known to give a rich source of natural anthelmintics. To date, there are no past studies covering the utilization of Balanites aegyptiaca fruit against cestodes. Yet, in light of the previously described anthelmintic activity (Shalaby et al. 2010; Shalaby et al. 2012, 2016) and its safety highlights, it is proposed in this investigation the Balanites extract's conceivable use against diphylidiasis. On the other hand, progressing levels of pet possession all over the world raises the significance of dogs. Besides, commercial and official uses of dogs in security and military increase the demand and attention to these animals. The prevalence of D. caninum in dogs worldwide ranged from 0.1 to $44 \%$ (Barutzki and Sohaper 2003; Dalimi et al. 2006), with rising the potential of dissemination of $D$. caninum eggs out the environment and risk-infecting diphylidiasis in human. The present study aimed to explore the role of $B$. aegyptiaca fruits against the adult $D$. caninum, evaluating its in vitro effects on adult worm tegument which is essential for the protective function, through microscopical approaches to define the mechanism of $B$. aegyptiaca fruits actions in the parasite.

\section{Materials and methods}

\section{Plant extract}

B. aegyptiaca fruits were obtained from the nearby markets in Aswan, Upper Egypt, and validated at the Herbarium of National Research Centre. Their methanolic extract was acquired as prescribed by Shalaby et al. (2010).

\section{Adult $D$. caninum}

Adult $D$. caninum were collected from the intestines of naturally infected stray dogs that were executed by the Egyptian police. After recovery, the worms were identified based on the macroscopic appearance of proglottids (Edwards and Herbert 1981) and washed in body-warm normal saline.

\section{In vitro treatment}

The entire worms, under sterile conditions in a laminar flow cabinet, were transferred to a normal saline solution containing $B$. aegyptiaca extract at a concentration of $240 \mu \mathrm{g} / \mathrm{ml}$. This concentration was chosen based on concentrations utilized in vitro with trials involving Toxocara vitulorum (Shalaby et al. 2012) and Paramphistomum microbothrium (Shalaby et al. 2016). A stock solution of methanolic extract at $10 \mathrm{mg} / \mathrm{ml}$ was prepared with a mixture of liquid paraffin and Tween 80 $(\mathrm{v} / \mathrm{v})$ for immediate use. Then, the entire worms were incubated for 12 and $24 \mathrm{~h}$ at $37^{\circ} \mathrm{C}$ in an atmosphere of $5 \%$ $\mathrm{CO}_{2}$. Solvent control worms were incubated for 12 and $24 \mathrm{~h}$ in normal saline solution containing $0.2 \%(\mathrm{v} / \mathrm{v})$ mixture of liquid paraffin and Tween 80 . Normal control worms were fixed immediately following the initial washing. Five worms were examined for each time period. The activity of worms was checked at 12- and 24-h incubation by visual observation and if necessary by physical excitation of the worms via gently agitating the culture media. The percent inhibition of motility of Balanites-treated cestodes was estimated using the following formula:

$$
\text { Inhibitory activity }=\frac{C-E}{C} \times 100
$$

where

$C$, mean number of control motile worms

$E$, mean number of exposed motile worms

\section{Statistical analysis}

Analysis of data was performed using the statistical program for the social sciences SPSS version 11. The significance of Balanites extract-induced inhibition in the motility of the cestodes was assessed using one-way analysis of variance (ANOVA) for each period of incubation.

\section{Light microscopy}

Following incubation, the gravid segments of Balanitestreated and untreated worms were cut into small, 5-mm pieces before being fixed at $10 \%$ buffered formol saline, and processed according to the method of Bancroft et al. (1996). The body wall of the gravid segments was studied and photographed using an Olympus CX41 microscope.

\section{Scanning electron microscopy (SEM)}

Following incubation, the anterior end of Balanitestreated and untreated worms was fixed intact for $12 \mathrm{~h}$ in a 3:1 mixture of $4 \%(\mathrm{w} / \mathrm{v})$ glutaraldehyde in $0.12 \mathrm{M}$ Millonig's buffer, $\mathrm{pH} 7.4$ and $1 \%$ aqueous osmium tetroxide. After this, the specimens were processed for SEM following a method previously reported (Shalaby et al. 2012).

\section{Results}

\section{Worm activity}

The control group exhibited no loss of activity during the whole period of incubation $(24 \mathrm{~h})$ and more sensitively reacted to changes in the surrounding conditions than the treated group. However, the latter group showed complete loss of motility after $24 \mathrm{~h}$ of incubation, even after being removed from the test medium and dipped in slightly warm normal saline and on gentle incitement; the paralyzed worms had not recovered their motility (Table 1). The inhibitory activity of Balanites extract on the motility for each period of incubation was significant $\left(P^{<} 0.005\right)$. To determine the mechanisms by which Balanites extract affected the adult worm activity, the possible tissue damage induced after treatment was 
Table 1 Effect of B. aegyptiaca fruits' extract on motility of adult D. caninum

\begin{tabular}{lll}
\hline Worms & \multicolumn{2}{l}{ Percentage of motility inhibition after incubation } \\
\cline { 2 - 3 } & $12 \mathrm{~h}$ & $24 \mathrm{~h}$ \\
\hline Control & 0.0 & 0.0 \\
Treated & $53.3 \pm 9.4$ & 100 \\
\hline
\end{tabular}

evaluated analyzing histological sections of the tegument of $D$. caninum gravid segments and structures of the tegumental surface.

\section{Light microscopic observations of the tegument transverse section}

On examining using light microscopy, the tegument of the solvent control worms showed normal features similar to that of the fresh control specimen. A short description of a portion of the vital tegumental features was important to evaluate the alterations resulting from Balanites extract treatment. The tegument showed an intensively stained syncytial layer, lay on a thick basement membrane of amorphous material containing granular inclusions. The basement membrane appeared to be continuous with the general filling material which lay between parenchymal cells of the interior of the proglottis. Beneath the basement membrane were two layers of smooth muscle, the outer occurring as circular bundles and the inner as longitudinal bundles. These layers were not massively developed and pursued by the subtegumentary layer of branching parenchymal cells (Fig. 1a-c).

After 12-h incubation in vitro with $240 \mu \mathrm{g} / \mathrm{ml} \mathrm{Bala-}$ nites extract, the tegument lost its normal aspect showing prominent wrinkles, accompanied by the appearance of focal areas of erosion and disruption of the muscle cells (Fig. 1d-f). These changes became more severe with the disruption of some areas of the outer tegumentary layer following 24-h incubation with Balanites extract. In these specimens, the tegument appeared to be swollen and showed extensive damage towards its surface and severe disruption of both muscle bundles and parenchymal tissues (Fig. 1g-i).

\section{Scanning electron microscopic observations of the adult worms}

On examining using SEM, no abnormal changes could be observed in the solvent control worms which showed normal body features. Its anterior end had a conical scolex with four suckers and an armed rostellum (Fig. 2a), forming the organs of attachment. Its body was an elongated ribbon-like structure (strobila) consisted of a chain of segments (proglottids). The segments budded from behind the scolex and were immature (Fig. 2b). As they were pushed back, they became mature and filled with eggs (Fig. 2c). The mature proglottid was longer that it was wide, and contained a common genital pore on either side (Fig. 2c inset).
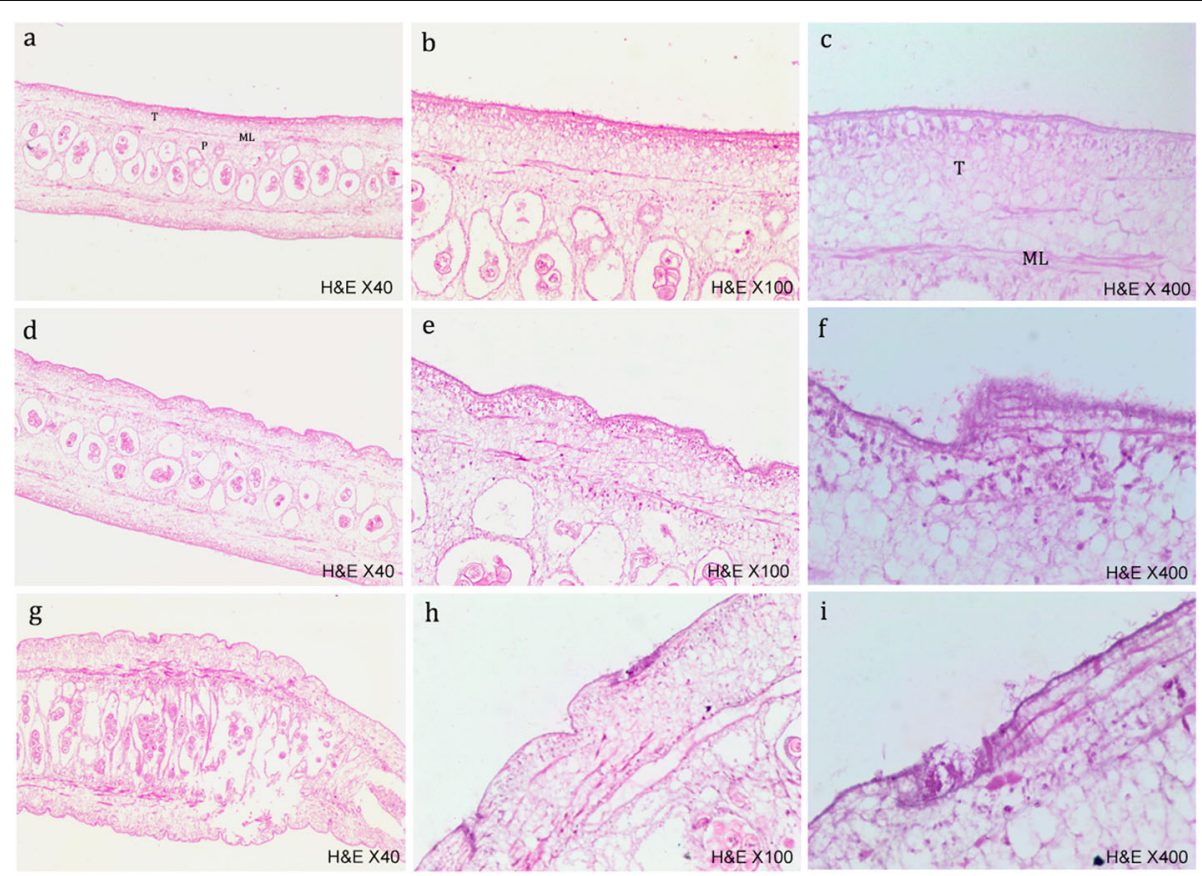

Fig. 1 Light micrographs of the tegument transverse section of adult D. caninum gravid segments. a-c Normal fresh cestodes. $\mathbf{d}$-f Following $12-$ h incubation with Balanites extract. Note the tegumental wrinkles and disruption of the muscle cells. g-i Following 24-h incubation with Balanites extract. In this specimen, the tegument appears to be swollen and shows extensive damage towards its surface. $T$ tegument, $M L$ muscle layer, $P$ parenchyma 


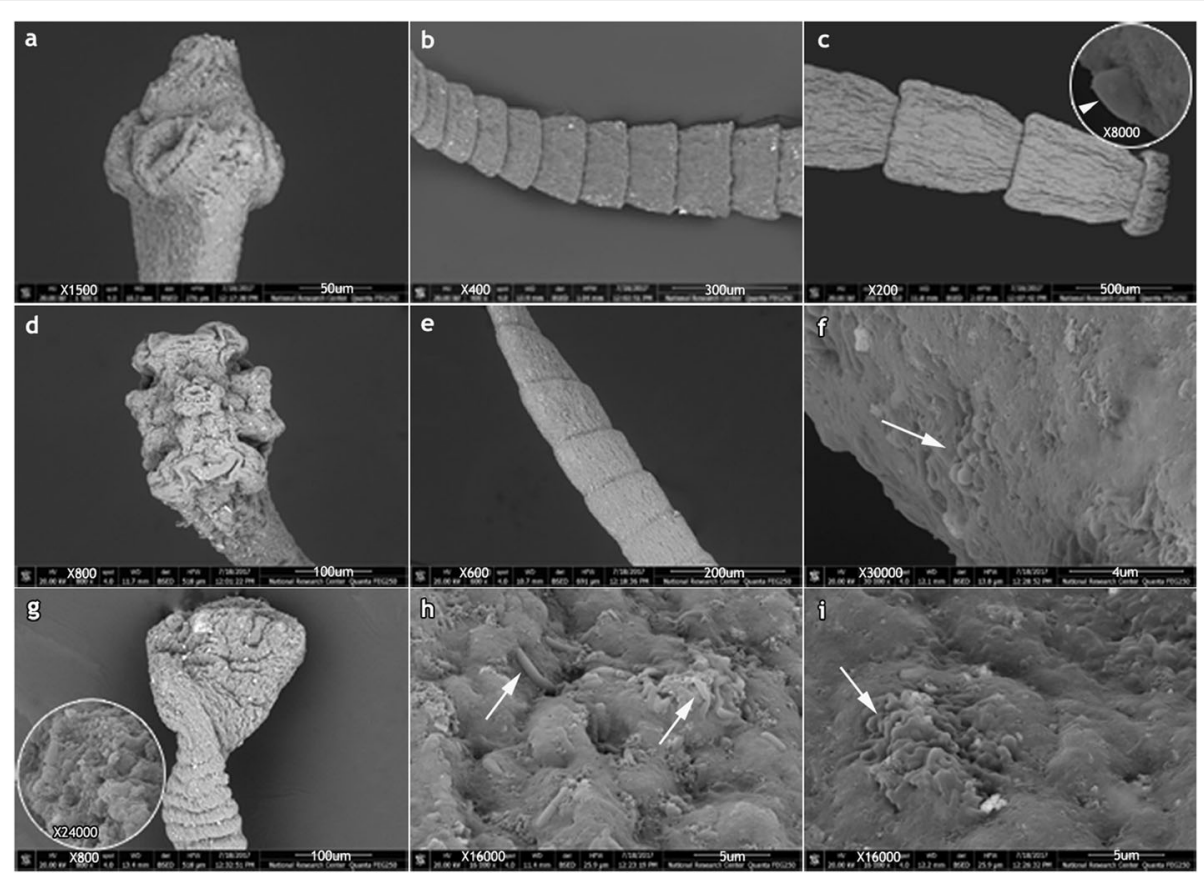

Fig. 2 Scanning electron micrographs (SEMs) of the adult D. caninum. a-c Normal fresh cestodes. Note the appearance of eggs' capsule at the common genital pore (head arrow). d-f Following 12-h incubation with Balanites extract. d SEM of the scolex showing severely folded tegument with distortion of both suckers and rostellum. e, $\mathbf{f}$ SEMs of the mature segments showing swollen tegument accompanied with areas of blebbing (white arrow). g-i Following 24-h incubation with Balanites extract. $\mathbf{g}$ SEM of the scolex showing severely distorted tegumental surface (inset) and extreme damage to both suckers and rostellum. $\mathbf{h}, \mathbf{i}$ SEMs of the mature segments revealing extensive tegumental damage and appearance of genital tubules (white arrows)

After 12-h incubation in vitro with $240 \mu \mathrm{g} / \mathrm{ml}$ Balanites extract, the tegumental changes concerned the scolex other than the proglottides. The scolex, in most of the examined specimens, showed severely folded tegument with distortion of both suckers and rostellum so that closure of their openings had occurred (Fig. 2d). In the proglottides, the tegument lost its normal aspect and appeared to be swollen than normal (Fig. 2e), accompanied with areas of blebbing occurred along its lateral margins (Fig. 2f). By 24-h incubation, and almost of all specimens examined, the changes in adult cestodes concerned the whole body surface. The scolex showed severely distorted tegumental surface and extreme damage to both suckers and rostellum that no structure could be recognized (Fig. 2g). In the proglottides, the distortion of the tegumental surface appeared to be more extensive than that described for the 12-h time period. The tegument might bear a number of lesions, where large blebs had burst leading to the appearance of the genital tubules (Fig. 2h, i).

\section{Discussion}

In this study, we explored the role of B. aegyptiaca fruits against the adult $D$. caninum, evaluating its in vitro effects on the adult worm tegument which is essential for the protective function. Our results show that Balanites extract exhibits cestocidal effect in vitro on $D$. caninum, and the tegument of the adult worm appears to be its primary target. This finding is supported by differences in extract response depending on the exposure period. After 12-h incubation in vitro with Balanites extract, the tegumental changes concerned the scolex other than the proglottides, while by 24 -h incubation, the changes in the adult cestodes concerned the whole body surface. The tegumental alterations were assessed by using both light and scanning electron microscopic studies. The distortion of the tegumental surface appeared to be the main changes induced by treatment with the tested extract. No reports of $B$. aegyptiaca effectiveness against cestodes are found. Yet, a previous in vitro study showed cuticular distortion of canine nematode, Toxocara canis, following its exposure to B. aegyptiaca fruit extract, with the result of decreased motility (Shalaby et al. 2018). On contrary, our finding revealed complete loss of cestode motility after 24-h exposure. The higher effectiveness to inhibit motility in this study may be attributed to the means by which the cestodes can nourish (Quiroz-Romero 2003), unlike the nematodes. Cestodes feed throughout the tegument, which means that the absorption surface of the extract may be higher, and because of the higher dose absorbed, the effective time is shorter. In this study, the tegumental alterations occurred in particular series in response to the 
exposure time, consisted of swelling, blebbing that was later ruptured, resulting in erosion and peeling of the tegument. Similar series of tegumental changes occurred in specimens of trematode, $P$. microbothrium, which affected ruminants, following their exposure to methanolic extract of B. aegyptiaca fruits (Shalaby et al. 2016). Such tegumental changes had been observed in other cestodes following their exposure to either synthetic or natural anthelmintics and seemed to be as a common countenance of drug-treated helminthes (Shalaby et al. 2018). Those changes might be referred to passive drug diffusion through the body wall of helminth (Moltier et al. 2003) and increased efforts on the portion of the helminth to spill and exchange the external tegumental membrane disrupted by the drug action (Stitt and Fairweather 1993). Previous studies had pointed to $B$. aegyptiaca fruits' methanolic extract as a potential source of natural anthelmintic because of its saponin constituents (Shalaby et al. 2016). Gnoula et al. (2007) confirmed the anthelmintic activity of a steroidal saponin isolated from $B$. aegyptiaca. The surface active and highly cytotoxic properties of saponins could be the most probable reason for the tegumental destructive effects of $B$. aegyptiaca fruits' methanolic extract on $D$. caninum adult worms.

\section{Conclusion}

Our study shows that B. aegyptiaca fruits' methanolic extract exhibits cestocidal effect in vitro on $D$. caninum, and the tegument of the adult cestode appears to be its primary target resulting in its damage. This damage would obviously disrupt the protective function of the tegument and facilitate expelling of the adult cestode from the dog's intestine. It seems that natural product obtained from Balanites extract may be a viable alternative for control of cestodes in dogs.

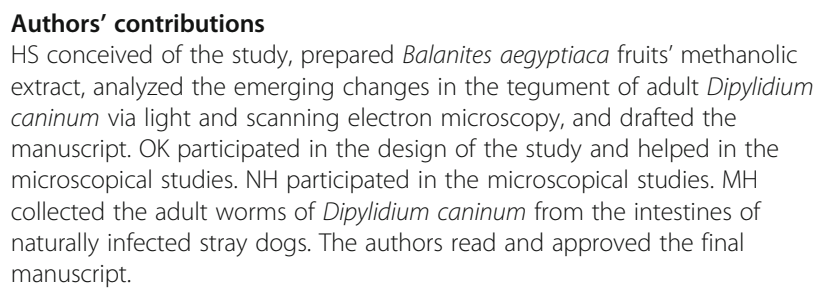

Funding

There is no funding.

Availability of data and materials

The tested B. aegyptiaca fruits are available at the nearby markets in Aswan, Upper Egypt, and validated at the Herbarium of National Research Centre.

Ethics approval and consent to participate

Not applicable.

Consent for publication

Not applicable.

Competing interests

The authors declare that they have no competing interests.
Received: 27 April 2019 Accepted: 31 March 2020

Published online: 10 April 2020

\section{References}

Bancroft JD, Stevens A, Wiley-Liss (1996) Theory and practice of histological techniques, 4th edn. Churchill Livingstone, New York, London, San Francisco, Tokyo

Barnett S, King S, Kok D, Luempert L (2013) Efficacy evaluation of flavored combination parasiticide tablets in a laboratory study for the removal of natural Dipylidium caninum (tapeworm) infections in dogs. Intern J Appl Res Vet Med 11:36-45

Barutzki D, Sohaper R (2003) Endoparasites in dogs and cats in Germany 19992002. Parasitol Res 90:S148-S150

Dalimi A, Sattari A, Motamedi G (2006) A study on intestinal helminthes of dogs, foxes and jackals in the western part of Iran. Vet Parasitol 142:129-133

Edwards GT, Herbert IV (1981) Some quantitative characters used in the identification of Taenia hydatigena, T. ovis, T. pisiformis and T. multiceps adult worms, and T. multiceps metacestodes. J Helminthol 55:1-7

Gnoula C, Guissou P, Duez P, Frederich M, Dubois J (2007) Nematocidal compounds from the seeds of Balanites aegyptiaca isolation and structure elucidation. Int J Pharmacol 3:280-284

Grandemange E, Claerebout E, Genchi C, Franc M (2007) Field evaluation of the efficacy and the safety of a combination of oxantel/pyrantel/praziquantel in the treatment of naturally acquired gastrointestinal nematode and/or cestode infestations in dogs in Europe. Vet Parasitol 145:94-99

Kopp SR, Kotze AC, McCarthy JS, Coleman GT (2007) High-level pyrantel resistance in the hookworm Ancylostoma caninum. Vet Parasitol 143:299-304

Moltier ML, Alvarez LI, Pis MA, Lanusse CE (2003) Transtegumental diffusion of benzimidazole anthelmintics into Moniezia benedeni: correlation with their octanol-water partition coefficients. Exp Parasitol 103:1-7

Quiroz-Romero H (2003) Parasitología y enfermedades parasitarias de los animales domésticos. Limusa, Mexico City, México

Shalaby H, El Namaky A, Kandil O, Hassan N (2018) In vitro assessment of Balanites aegyptiaca fruit methanolic extract on the adult Toxocara canis. Iran J Parasitol. 13:643-647

Shalaby H, Nasr S, Farag T (2016) Tegumental effects of methanolic extract of Balanites aegyptiaca fruits on adult Paramphistomum microbothrium (Fischoeder 1901) under laboratory conditions. Iran J Parasitol 11:396-405

Shalaby HA, El Namaky AH, Khalil FA, Kandil OM (2012) Efficacy of methanolic extract of Balanites aegyptiaca fruit on Toxocara vitulorum. Vet Parasitol 183: 386-392

Shalaby MA, Moghazy FM, Shalaby HA, Nasr SM (2010) Effect of methanolic extract of Balanites aegyptiaca fruits on enteral and parenteral stages of Trichinella spiralis in rats. Parasitol Res 107:17-25

Stitt AW, Fairweather I (1993) Fasciola hepatica: tegumental surface changes in adult and juvenile flukes following treatment in vitro with the sulphoxide metabolite of triclabendazole (Fasinex). Parasitol Res 79:529-536

Szwaja B, Romanski L, Zabczyk M (2011) A case of Dipylidium caninum infection in a child from the southeastern Poland. Wiadomosci Parazytologiczne 57: 175-178

Wani ZA, Allaie IM, Shah BM, Raies A, Athar H, Junaid S (2015) Dipylidium caninum infection in dogs infected with fleas. J Parasit Dis 39:73-75

Yangco BG, De Lerma C, Lyman GH, Price DL (1987) Clinical study evaluating efficacy of praziquantel in clonorchiasis. Antimicrob Agents Chemother 31: $135-138$

\section{Publisher's Note}

Springer Nature remains neutral with regard to jurisdictional claims in published maps and institutional affiliations. 